\title{
Galilean-Like Transformation Allowed by General Covariance and Consistent with Special Relativity
}

\author{
Frank R. Tangherlini \\ P.O. Box 928211, San Diego, CA 92192, USA \\ Email: frtan96@gmail.com
}

Received 12 December 2013; revised 11 January 2014; accepted 13 February 2014

Copyright (C) 2014 by author and Scientific Research Publishing Inc.

This work is licensed under the Creative Commons Attribution International License (CC BY). http://creativecommons.org/licenses/by/4.0/

\section{(c) (i) Open Access}

\begin{abstract}
As shown earlier, a linear transformation with the same form for the spatial coordinates as the Lorentz transformation (LT), and that allows for time dilation, but leaves simultaneity invariant instead of the one-way speed of light, predicts the same results as the LT for the usual tests of special relativity. Such a transformation is allowed by general covariance. A complementarity between the invariance of the one-way speed of light and the invariance of simultaneity is discussed. Using this transformation, interpreted as involving external synchronization, it is shown that two frames moving uniformly with equal and opposite velocities, $v$ and $-v$ relative to a third inertial frame, in which clocks are synchronized so that the one-way speed of light is $c$, can be related by a Galilean-like transformation with a relative velocity $2 v /\left(1-(v / c)^{2}\right)$. These transformations do not form a group, hence the term "pseudo-Galilean" is used to distinguish them from the Galilean transformations. An analogy with the Sagnac effect is discussed, and consistency with the LT for stellar aberration, and the Doppler effect is shown. Implication of the above complementarity for the possible unification of quantum theory and gravitation is briefly discussed, as well as the inferred physical significance of general covariance.
\end{abstract}

\section{Keywords}

General Covariance; Transformations; Synchronization; Complementarity

\section{Introduction}

It was shown in the author's Ph.D. dissertation [1] that a linear transformation that has the same form for the 
spatial coordinates as the Lorentz transformation (LT), but differs for the time transformation, inasmuch as it keeps simultaneity invariant between the two frames, while preserving the relativistic time dilation, predicts the same results as special relativity (SR) for the out-and-back speed of light, as well as for the other numerous tests of SR. It was pointed out that the use of such a transformation that does not leave the Minkowski metric invariant can be justified on the basis of Einstein's principle of general covariance. For a study of Einstein's work leading to general covariance see Stachel [2], and for further discussion see, e.g., Pauli [3], Rindler [4], and Weinberg [5]. It was also shown in [1] that upon transforming the time coordinate in an LT frame to the time coordinate of this alternative transformation by means of a local time transformation, the Schrödinger state function undergoes a unitary transformation, so that the observables of the two different transformations are the same. More recently, the author [6] showed that for a suitable generalization of the canonical commutation relations to include time, they remain invariant under all linear, homogeneous space-time transformations, an invariance which therefore includes the transformations studied here.

The main purpose of this work is to show, with the aid of this transformation, given in Section 2, that two frames moving uniformly with equal and opposite velocities $\mathbf{v}$ and $-\mathbf{v}$ relative to a third inertial frame in which clocks have been synchronized in accordance with SR, so that the one-yay speed of light is $c$ in all directions, are related by a Galilean-like transformation with relative velocity $2 v /\left(1-(v / c)^{2}\right)$, when the clocks are synchronized externally with those in that third frame. This external synchronization is in contrast to the treatment in [1], in which the clocks for the transformation were assumed to be synchronized by means of hypothetical signals propagating instantaneously with respect to the hypothetical ether frame. As a possible means to realizing such signals, this led in [1] to an anticipation of tachyons, as they were called and later investigated by Feinberg [7]. See also the related writings of Schmidt [8] [9], Bilaniuk et al. [10], Tanaka [11], and Terletskii [12]. For reviews of tachyons, see, Recami [13] and Fröman [14]. However, later, with a view to eliminating unnecessary, and possibly incorrect hypotheses (e.g., since tachyons have imaginary mass, a mass operator whose eigenvalues would give the masses of the elementary particles would not be Hermitian, in conflict with quantum mechanics), an experimentally realizable alternative to such hypothetical faster-than-light methods of synchronization was introduced by the author [15], which he had earlier rejected as unjustified [16]. This method involves external synchronization directly with an arbitrary inertial frame. For the purposes here, an inertial frame is one in which space-time coordinates can be chosen so that Newton's first law holds for a test body so that, in the absence of an external force, $\mathrm{d}^{2} x^{\mu} / \mathrm{d} s^{2}=0$, all the Christoffel symbols vanish, and hence the $g_{\mu v}$ are constants. Such coordinates are used in what follows; they are all transformable into one another by linear transformation, and are clearly inertial.

Because of the utilization of external synchronization, the transformation will be referred to here as the externally synchronized transformation (EST) rather than the absolute Lorentz transformation (ALT) used in [1]. The transformation is referred to by a variety of other names in the literature, frequently, the "Tangherlini transformation" or just the "TT." In addition to tests of the transformation described in [1], extensive discussion is given in Mansouri and Sexl [17]. Although an application to Maxwell's equation is in [1], it was for motion along one axis; Chang [18] extended this to motion along an arbitrary axis. Further application to Maxwell's equations is given by the author in [19]. For a study of synchronization in special relativity, see, e.g., Sjödin [20]. For interesting critiques of the earlier formulation based on instantaneous signals, see Cavaleri and Spinelli [21], and Spavieri [22]. For a discussion of the transformation's inertial properties, and other topics, see Selleri [23] [24]. For a novel application that compares the LT with the EST for the case of relativistic fluid media, see Cavalleri and Tonni [25], and Tonni [26]. For revisions of [25] [26], see Cavalleri et al. [27]. For additional references to the transformation, and comparison with alternative linear space-time transformations, see Malykin and Malykin [28]. Interestingly, they found that an earlier presentation of the transformation, albeit without reference to the transverse coordinates, is due to Eagle [29] [30]. However, his synchronization method was based on the erroneous idea that instantaneous synchronization can be achieved by rotating a cylinder or "spindle", and hence does not allow for the fact that torsional waves, or elastic waves in material media more generally, propagate with finite speeds not exceeding that of light. There was no attempt to justify the transformation from the standpoint of general covariance, in view of his rejection of general relativity as well as special relativity [31]. The historical motivation for the author's work was originally based on the divergences in quantum electrodynamics, as well as general covariance, and is described in the 2009 preface to the dissertation in [32], where there is also described the application to Reichenbach synchronization [33] when represented by a local time transformation, as given in Anderson et al. [34]. This is omitted here for brevity, as is also discussions about the ether, for the 
latter see [15] [32]. In Section 3, a theoretical justification for using external synchronization will be given that is suggested by general covariance, restricted here, however, to linear, homogeneous transformations. Also included in the discussion will be the possible experimental realizations of such an external synchronization, which will include reference to the Cosmic Microwave Background Radiation. In Section 4, analogies of possible measurements made with the EST with the relativistic Sagnac effect for vacuum and optical fiber transmission will be presented. In Section 5, the Galilean-like transformation, which will be called the "Pseudo-Galilean transformation" (PGT), will be derived, and some of its properties discussed. Also, the line element for the EST and its transformation under the PGT will be given. In Section 6, it will be shown that the PGT makes the same prediction for stellar aberration as the Lorentz transformation. In Section 7, there is an application of the PGT to the Doppler effect that demonstrates again its agreement with the LT. In Section 8, it will be pointed out that the transformation brings out that a complementarity exists between maintaining invariance of simultaneity and maintaining invariance of the one-way speed of light in a space-time coordinate transformation, and its possible implication for the unification of gravity and quantum theory, as well as further discussion of general covariance. In Section 9, there are concluding remarks that include possible further applications of the PGT to particle physics.

\section{The Externally Synchronized Transformation}

As given in [1], and later in [15] [16], the transformation for uniform motion in the x-direction with velocity $v$ relative to an inertial frame in which clocks have been synchronized in accordance with SR is

$$
\begin{gathered}
x^{\prime}=\gamma(x-v t), \quad y^{\prime}=y, \quad z^{\prime}=z, \\
t^{\prime}=\gamma^{-1} t,
\end{gathered}
$$

where $\gamma=\left(1-(v / c)^{2}\right)^{-1 / 2}$. The transformation for the spatial coordinates is the same as for the LT, while the time transformation, in contrast to the LT, keeps simultaneity invariant. Thus, if two events are simultaneous in the unprimed frame $\Delta t=0$, they are also simultaneous in the primed frame $\Delta t^{\prime}=0$. As mentioned in Section 1 , in contrast with the earlier interpretation that hypothesized superluminal synchronization, here the time transformation is obtained by synchronizing the clocks in the primed frame directly with those in the unprimed frame. This can be accomplished in a way that is capable of experimental implementation at present. Assume the unprimed frame is a railroad station (taken as an inertial frame after suitable corrections), and along the platform parallel to the tracks there is fixed a row of identical clocks that have been synchronized by Einstein's method, or by slow transport, so that the one-way speed of light is $c$. Assume further that on a train passing with constant speed $v$ in the positive $x$-direction, there is a row of clocks identical to those in the station, but unsynchronized, and that there are also transverse-oriented, electro-mechanical devices that enable clocks on the train to receive signals from clocks on the station. Then when clocks on the station read $t=0$, this is communicated to the train's clocks as they pass by, setting them to zero (correcting for the transverse travel time), after which the clocks on the train are allowed to run freely at their own rate. Hence, in accordance with (2), they will run more slowly than clocks in the station, but at the same rate as clocks synchronized according to the LT. Since the inverse of the EST is given by

$$
\begin{gathered}
x=\gamma^{-1} x^{\prime}+v \gamma t^{\prime}, y=y^{\prime}, z=z^{\prime}, \\
t=\gamma t^{\prime},
\end{gathered}
$$

the inverse of the EST is not of the same form as the EST, and clocks in the station run faster than clocks on the train, and rods on the station are longer than rods on the train, when measured by clocks and rods on the train. This contrasts with the LT for which there is complete reciprocity, and is due to the fact that the ESTs do not form a group, even for velocity boosts along the same axis, unlike the LTs which do. Likewise, the EST does not keep the one-way speed of light invariant since

$$
\mathrm{d} x^{\prime} / \mathrm{d} t^{\prime}=\gamma^{2}(\mathrm{~d} x / \mathrm{d} t-v) .
$$

Hence, in the forward direction, the speed of light is $c /(1+(v / c))$, while in the rearward direction the speed is $c /(1-(v / c))$, but importantly, the total travel time to go out and back for a distance $L^{\prime}$ is just $2 L^{\prime} / c$. Thus the EST keeps the out-and-back speed of light invariant in agreement with the LT, and to be sure, the Michel- 
son-Morley experiment. Although in the discussion above, the station clocks have been synchronized so that the one-way speed of light is $c$, alternatively, because of the relativity principle, one could have synchronized clocks on the train so that the one-way speed of light is $c$, and synchronized the clocks in the station with those on the train, and then used the EST with $v \rightarrow-v$ to transform to the station.

\section{External Synchronization and Simultaneity}

Although the transformation given in Equations (1) and (2) is obviously allowed mathematically according to Einstein's principle of general covariance, one might wonder whether there is a more physical justification for it? A possible answer to this question emerges from the following consideration about the relativity of simultaneity occasioned by the LT for the time coordinate which in the transformed frame will be denoted by $t_{L}$, and for coordinate differences is given by the standard relation

$$
\Delta t_{L}=\gamma\left(\Delta t-\left(v / c^{2}\right) \Delta x\right)
$$

As Einstein first recognized, if two events are spatially separated in the unprimed frame, $\Delta x \neq 0$, and are simultaneous in that frame, $\Delta t=0$, they are not simultaneous in the moving frame, $\Delta t_{L} \neq 0$. But while this follows mathematically, it cannot be demonstrated empirically, and hence scientifically, unless there is an exchange of information between the two frames, and this occasions the opportunity for external synchronization. In the general situation, say a frame in uniform motion through outer space, one can replace the railroad station frame by the Cosmic Microwave Background Radiation (CMBR), which was found by COBE, as described in Smoot et al. [35], to be uniform to about one part in $10^{5}$, as the external frame with which to synchronize. With the aid of the CMBR, one can establish the EST time by synchronizing clocks in the space vehicle frame internally according to SR, and then determining with onboard antennas the velocity of the frame relative to the CMBR from the dipole in the radiation, found originally by Conklin [36] and Henry [37]. With this information and suitable alignment of the coordinate system, the EST time is given by

$$
t^{\prime}=t_{L}+\left(v / c^{2}\right) x_{L}
$$

It is of course assumed one is working in a patch of space-time so sufficiently small that one can treat it as flat, and that the CMBR is perfectly uniform.

Returning to the case of clocks on a train moving uniformly with speed $v$ through the station, treated as an inertial frame, it is shown in [1] that when a clock on the train is slowly displaced a distance $\delta x^{\prime}$ in the direction of the train's motion relative to a similar clock on the train that remained at rest, so that its velocity changes by $\delta v$, the change in the time of the displaced clock $\delta t^{\prime}$ calculated using Equations (1) and (2), is given by

$\delta t^{\prime}=-\left(v / c^{2}\right) \delta x^{\prime}$, and hence the displaced clock acquires the Lorentz transformation for the time, since $t_{L}=t^{\prime}-\left(v / c^{2}\right) x^{\prime}$. More generally, the result holds independently of the displacement direction. For an experimental verification to high accuracy that the slow transport of a clock in an inertial frame yields a synchronization consistent with Einstein's method of synchronization, and hence also supports the above analysis based on the EST, see Wolf and Petit [38].

\section{Analogies of the EST with the Sagnac Effect}

There are interesting analogies with the Sagnac effect [39] [40] that follow from measurements made with the EST, both for vacuum, and for the case of a refractive medium, that will now be discussed. Since the speed of light in the primed frame in general is different in the out and back directions, the difference of the two travel times in general does not vanish, unlike the case for the LT. For the simplest case, in which light travels in a vacuum out and back along the $x^{\prime}$-axis a distance $D^{\prime}$, one has for the time difference

$$
\Delta t^{\prime}=t^{\prime}(\rightarrow)-t^{\prime}(\leftarrow)=2 v D^{\prime} / c^{2}=2 v \gamma D / c^{2},
$$

where use has been made of the fact that for the EST, in contrast to the LT, the length $D$ in the unprimed frame appears longer in the primed frame, so that $D^{\prime}=\gamma D$, as remarked following (4). Thus, because of the external synchronization, observers in $S^{\prime}$, e.g. the train, can determine their speed relative to $S$, the station. This result lends itself to a direct comparison with the Sagnac effect, although his experiment involved interferometry, whereas here only time differences are involved, and light is treated solely in the geometrical optics limit; also 
his experiment was non-relativistic, in contrast with the analysis here, in which it is also assumed that the path is exactly circular, which was not the case for his experiment. For reviews, see Post [41], Anderson et al. [42], Malykin [43], and Ghosal et al. [44].

Assume one has a disk of radius $R$ as measured in an inertial frame $S$, in which clocks have been synchronized so that the speed of light is $c$ all directions, so that a vacuum is assumed, and also that the disk is rotating in the $x-y$ plane, with angular velocity $\omega$, as measured in $S$, in the counter-clockwise direction, when viewed from above. From a source of light located on the periphery of the rotating disk, light beams are sent by means of mirrors around the periphery in counter-clockwise ( $c c w)$ and clockwise $(c w)$ directions. The difference in times of arrival when they return to the source, as measured by clocks at rest in $S$, is given by

$$
\Delta t=t(c c w)-t(c w)=4 \pi R^{2} \omega / c^{2}\left(1-(R \omega / c)^{2}\right) .
$$

Customarily, upon neglect of the higher-order term in the denominator, the above is written as $4 A \omega / c^{2}$, where $A=\pi R^{2}$ is the area of the circuit. This is the standard, non-relativistic expression for the Sagnac effect for a time measurement. But this form is not convenient for the comparison given here. If, located at the light source on the disk periphery, there is also a clock that registers the difference of arrival times of the two beams, then because of the time dilation factor $\gamma^{-1}=\left(1-(R \omega / c)^{2}\right)^{1 / 2}$, the proper time difference $\Delta \tau$ that the clock will read is

$$
\Delta \tau=4 \pi R^{2} \omega \gamma / c^{2} .
$$

In comparison with Equation (8), if $2 \pi R \gamma$ is identified with $\gamma D$ and $R \omega$ with $v$, there is perfect agreement. Note that $\Delta t^{\prime}$ in (8) is the proper time interval for a clock at rest in $S^{\prime}$, and since the transformation to the clock on the periphery of the rotating disk for the time interval $\Delta t$ in $S$ is $\Delta \tau=\left(1-(\omega R / c)^{2}\right)^{1 / 2} \Delta t$, the analogy with the EST is exact. Clearly, the above proper time difference behaves as if the rotating disc were externally synchronized to the inertial frame relative to which it is rotating. Indeed it is, because in the circular case the clock at B, that in the linear case is separated from the clock at A, in the present case is at the same location as A, for a closed path around the circle. Hence if the clock at A is synchronized externally, the clock at B can be as well. Indeed, the clock at B can be the same clock as that at A, in contrast with the linear case for the train. Further, since time differences are involved, the clock in the rotating frame does not have to be set to agree initially with a clock in the inertial frame, since they necessarily agree to within a constant, which will be the same for clocks at B and A, and hence cancel when the time difference is taken.

The above discussion assumes that light travels through a vacuum, but the analogy with the EST holds when light is constrained to be traveling through an optical fiber, and furthermore, the analogy makes an interesting prediction that is borne out by observation.

Suppose that on the uniformly moving train the two sets of separated clocks are connected by an optical fiber of index of refraction $n$. The EST clocks are related to the LT clocks by the local time transformation given in (7) so that $t^{\prime}=t_{L}+\left(v / c^{2}\right) x_{L}$, with $x_{L}=x^{\prime}$. Then, by the relativity principle, the out and back times associated with the LT, although longer, are still equal as they were for the vacuum case, so that $t_{L}(\rightarrow)=t_{L}(\leftarrow)=n D^{\prime} / c$, and hence they continue to cancel, so that when one calculates $\Delta t^{\prime}$, it remains the same as in (8), and hence does not involve the index $n$. Then, if the analogy continues to hold when the Sagnac effect is studied for the case in which light travels around its circular path on the rotating disk confined to an optical fiber, or an equivalent refractive medium, the effect should not depend on the index $n$, and this is indeed the case, as first recognized by Harzer [45] in a re-examination of the dissertation of Harress [46]. In addition to the above reviews and analysis, subsequent critique of earlier work is by Arditty and Lefèvre [47] that employs Maxwell's equations in a medium.

One can of course obtain the independence of the time difference on $n$ directly by introducing cylindrical coordinates, and noting that in the plane of the disk, the line element takes the form $d s^{2}=c^{2} \mathrm{~d} t^{2}-r^{2} \mathrm{~d} \phi^{2}$. Then for a circular path of radius $R$, the line element becomes $\mathrm{ds} s^{2}=c^{2} \mathrm{~d} t^{2}-R^{2} \mathrm{~d} \phi^{2}$, consequently one can make what might be called a circular Lorentz transformation (CL) from the rest frame to the rotating frame of the disk, given by, $\phi_{C L}=\gamma(\phi-\omega t), \quad t_{C L}=\gamma\left(t-\left(R^{2} \omega / c^{2}\right) \phi\right)$, so that upon transforming to the rotating frame, the line element becomes $\mathrm{ds} s^{2}=c^{2}\left(\mathrm{~d} t_{C L}\right)^{2}-R^{2}\left(\mathrm{~d} \phi_{C L}\right)^{2}$, and is clearly of the same form as in the rest frame, thus manifesting a circular relativity principle in which the speed of light both clockwise and counterclockwise is the 
same when $t_{C L}$ is used to measure time. It is therefore reasonable to assume that because of this circular relativity, the speed of light in the optical fiber when measured in these new coordinates in both directions is $c / n$. Then, using the expressions for relativistic relative velocity as seen in the rest frame, one has for the counter-clockwise direction, $R \mathrm{~d} \phi / \mathrm{d} t=(c / n+R \omega) /(1+(R \omega / c n))$ and for the clockwise direction

$R \mathrm{~d} \phi / \mathrm{d} t=(c / n-R \omega) /(1-(R \omega / n c))$. Then when one calculates the time difference in the rest frame for the two oppositely traveling light signals to complete their journey, one finds the standard result that it is independent of $n$. Indeed, expressions such as the above for the relativistic relative velocity are customarily justified by considering the infinite set of Lorentz transformations tangent to the rotating disk. To generate $t_{C L}$ experimentally, one slowly moves a clock $\mathrm{B}$ around the circle of radius $\mathrm{R}$ in either direction from the initial position where there is a clock $\mathrm{A}$, back to the initial position; this is entirely analogous to generating $t_{L}$ on the uniformly moving train.

However, to understand why the train analogy works, one introduces instead of $t_{C L}$ the externally synchronized time $t^{\prime}=\gamma^{-1} t$, along with $\phi^{\prime}=\phi_{C L}$. Since, similar to the linear case, one has $t^{\prime}=t_{C L}+\left(R^{2} \omega / c^{2}\right) \phi_{C L}$, one sees immediately that because the travel times around the disk in CL time satisfy $t_{C L}(c c w)=t_{C L}(c w)$, the difference in travel times does not depend on $n$. It is given by

$$
\Delta t^{\prime}=2\left(R \omega / c^{2}\right) R \Delta \phi_{C L}
$$

where $R \Delta \phi_{C L}$ is the circumference of the disk measured in the rotating frame. To determine $R \Delta \phi_{C L}$, one has that in the rest frame the circumferential angle measured for $\Delta t=0$ is $2 \pi$ and hence $\Delta \phi_{C L}=\gamma 2 \pi$, so that $\Delta t^{\prime}=4 \pi R^{2} \omega \gamma / c^{2}$, in agreement with the vacuum case. The line element in terms of $t^{\prime}$ and $\phi^{\prime}$ is $\mathrm{d} s^{2}=c^{2}\left(\mathrm{~d} t^{\prime}\right)^{2}-2 R^{2} \omega \mathrm{d} t^{\prime} \mathrm{d} \phi^{\prime}-\left(1-\left(R^{2} \omega^{2} / c^{2}\right)\right) R^{2}\left(\mathrm{~d} \phi^{\prime}\right)^{2}$. A similar line element for the EST is given in the next section, see Equation (25).

\section{The Pseudo-Galilean Transformation}

Assume there are two inertial frames $S^{\prime}$ and $S^{\prime \prime}$ moving with speed $v$ in the positive and negative $x$-directions, respectively, relative to a third inertial frame $S$ in which clocks have been synchronized so that the speed of light is $c$ in all directions. Let the clocks in $S^{\prime}$ and $S^{\prime \prime}$ be synchronized externally with those in $S$. The corresponding ESLT from $S$ to $S^{\prime}$ is given in (1) and (2), while that from $S$ to $S^{\prime \prime}$ is obviously the same as that from $S$ to $S^{\prime}$, with $v \rightarrow-v$, and it is given here explicitly for later reference

$$
\begin{gathered}
x^{\prime \prime}=\gamma(x+v t), y^{\prime \prime}=y, z^{\prime \prime}=z, \\
t^{\prime \prime}=\gamma^{-1} t .
\end{gathered}
$$

Upon substituting the inverse of this transformation, which is given by Equations (3) and (4) with $v \rightarrow-v$, into (1) and (2), the transformation relating $S^{\prime}$ to $S^{\prime \prime}$ is

$$
\begin{gathered}
x^{\prime}=x^{\prime \prime}-2 v \gamma^{2} t^{\prime \prime}, y^{\prime}=y^{\prime \prime}, z^{\prime}=z^{\prime \prime}, \\
t^{\prime}=t^{\prime \prime} .
\end{gathered}
$$

This transformation is exactly of the same form as a Galilean transformation for which the relative velocity $u$ of $S^{\prime}$ with respect to $S^{\prime \prime}$ is given by

$$
u=2 v \gamma^{2}
$$

in which $u$ ranges, analogous to the Galilean transformation, $-\infty<u<\infty$, for $-1<v / c<1$.

The expression for $u$ in (15) follows from (11) and (12), since $\mathrm{d} x^{\prime \prime} / \mathrm{d} t^{\prime \prime}=\gamma^{2}(\mathrm{~d} x / \mathrm{d} t+v)$, and also that $\mathrm{d} x / \mathrm{d} t=v$ for the motion of $S^{\prime}$ with respect to $S$.

This Galilean-like transformation, that keeps simultaneity invariant, indicates that when the lengths of similar rods and the rates of similar clocks are compared between the two frames, they are the same, in contrast with the prediction of the Lorentz transformation, which would lead observers in either frame to find the other frame's rods are shorter in the direction of motion, their clocks keep time more slowly, and simultaneity is relative. This resolves the apparent paradox that is often raised concerning this situation, as discussed e.g., in Bridgman [48]. The frames $S^{\prime}$ and $S^{\prime \prime}$ can be represented by two trains traveling in opposite directions with the same speed relative to the station $S$. Because of the symmetry between the states of the two trains as seen from the station, 
bearing in mind the isotropy of space, and assuming equal preparation, there is no dynamical way that similar rods and clocks on the one train could be physically different than those on the other train, hence the contraction and dilation given by the LT, although experimentally observable, is nevertheless an appearance that results from the use of internal synchronization.

However, some caution is necessary in using the transformation, since unlike the Galilean transformation, the relative velocity of $S^{\prime}$ with $S^{\prime \prime}$, is not $2 v$, but rather, $2 v \gamma^{2}$. If the Galilean-like transformation is written as $G\left(2 v \gamma^{2}\right)$, then as follows from (13), it has one of the properties of forming a group, since its inverse has the same form

$$
G^{-1}\left(2 v \gamma^{2}\right)=G\left(-2 v \gamma^{2}\right)
$$

On the other hand, the product rule is not obeyed, since

$$
G\left(2 v_{1} \gamma_{1}^{2}\right) G\left(2 v_{2} \gamma_{2}^{2}\right)=G\left(\frac{1-\left(v_{1} v_{2} / c^{2}\right)}{1+\left(v_{1} v_{2} / c^{2}\right)} 2 V \gamma_{V}^{2}\right),
$$

in which $V$ is the relativistic sum of velocities, $V=\left(v_{1}+v_{2}\right) /\left(1+\left(v_{1} v_{2} / c^{2}\right)\right)$, and $\gamma_{V}=\gamma(V)$. Hence, as with the EST, these transformations do not form a group, even when the boost is along the same axis as the original velocity. Thus it seems appropriate to describe the transformation as the pseudo-Galilean transformation (PGT), so as to avoid confusion with the Galilean transformations which, as is well-known, do form a group.

When the velocities are not along the $x$-axis, the EST is obtained by using the vector expression for the LT for the spatial coordinates, but making the appropriate change for the transformation of the time coordinate. The EST in vector form, is then given by

$$
\begin{gathered}
\boldsymbol{r}^{\prime}=\boldsymbol{r}+\boldsymbol{v}(\boldsymbol{r} \cdot \boldsymbol{v}) v^{-2}(\gamma-1)-\gamma \boldsymbol{v} t, \\
t^{\prime}=\gamma^{-1} t,
\end{gathered}
$$

Similar equations relate $S^{\prime \prime}$ to $S$ upon setting $\boldsymbol{v} \rightarrow-\boldsymbol{v}$. After subtraction of the equations relating $S^{\prime \prime}$ to $S$ from (18) and (19), the vector form of the PGT is given by

$$
\begin{gathered}
\boldsymbol{r}^{\prime}=\boldsymbol{r}^{\prime \prime}-2 \boldsymbol{v} \gamma^{2} t^{\prime \prime}, \\
t^{\prime}=t^{\prime \prime} .
\end{gathered}
$$

Equation (20) can alternatively be written in tensor form as

$$
x^{\prime i}=x^{\prime \prime i}-2 v_{i} \gamma^{2} t^{\prime \prime}
$$

Written in this way, there is of course no restriction to three spatial dimensions, so the spatial coordinate dimensionality can have the range $i=1, \cdots, n$. The local time transformations connecting $S^{\prime}$ and $S^{\prime \prime}$ with their corresponding Lorentz frames, with sum over repeated indices, are

$$
\begin{gathered}
t_{L}^{\prime}=t^{\prime}-\left(v_{i} / c^{2}\right) x^{\prime i}, \quad x_{L}^{\prime i}=x^{\prime i}, \\
t_{L}^{\prime \prime}=t^{\prime \prime}+\left(v_{i} / c^{2}\right) x^{\prime \prime i}, \quad x_{L}^{\prime \prime i}=x^{\prime \prime i} .
\end{gathered}
$$

Upon inserting these local time transformations into the corresponding Minkowskian line elements for the two Lorentz frames, one has

$$
\begin{aligned}
& \mathrm{d} s^{2}=c^{2} \mathrm{~d} t^{\prime 2}-2 v_{i} \mathrm{~d} x^{\prime i} \mathrm{~d} t^{\prime}-\left(\delta_{i j}-c^{-2} v_{i} v_{j}\right) \mathrm{d} x^{\prime i} \mathrm{~d} x^{\prime j}, \\
& \mathrm{~d} s^{2}=c^{2} \mathrm{~d} t^{\prime \prime 2}+2 v_{i} \mathrm{~d} x^{\prime \prime i} \mathrm{~d} t^{\prime \prime}-\left(\delta_{i j}-c^{-2} v_{i} v_{j}\right) \mathrm{d} x^{\prime \prime i} \mathrm{~d} x^{\prime \prime} .
\end{aligned}
$$

After transforming Equation (25) by setting $\mathrm{d} t^{\prime}=\mathrm{d} t^{\prime \prime}$ from (21), and setting $\mathrm{d} x^{\prime i}=\mathrm{d} x^{\prime \prime i}-2 v_{i} \gamma^{2} \mathrm{~d} t^{\prime \prime}$ from Equation (22), one obtains the line element in Equation (26). In verifying this, summation over repeated indices is understood, so that, e.g., $-2 v_{i} \mathrm{~d} t^{\prime \prime}\left(-2 v_{i} \gamma^{2} \mathrm{~d} t^{\prime \prime}\right)=4 v^{2} \gamma^{2} \mathrm{~d} t^{\prime 2}$, and also one has that

$-\left(\delta_{i j}-c^{-2} v_{i} v_{j}\right)\left(-2 v_{j} \gamma^{2} d t^{\prime \prime}\right) \mathrm{d} x^{\prime \prime i}=2 v_{i} \mathrm{~d} t^{\prime \prime} \mathrm{d} x^{\prime \prime i}$, a term that will occur again with a different dummy index, and finally, $-\left(\delta_{i j}-c^{-2} v_{i} v_{j}\right)\left(4 v_{i} v_{j} \gamma^{4} \mathrm{~d} t^{\prime \prime 2}\right)=-4 v^{2} \gamma^{2} \mathrm{~d} t^{\prime \prime 2}$, which just cancels the same term that occurred above with a 
plus sign. Note that since the only difference between the $S^{\prime \prime}$ frame and the $S^{\prime}$ frame is that they are traveling with equal and opposite velocities relative to the rest frame $S$, by symmetry considerations, Equation (26) should also follow from Equation (25) by replacing $v_{i}$ by $-v_{i}$, which is indeed the case.

If one asks for the inverse to the metric in Equation (25), or in Equation (26), one finds a surprising result: it is the metric that would result if one had transformed the Minkowski metric from the $S$ frame by a standard Galilean transformation (GT). Thus, upon making the Galilean transformation, $x_{G}^{i}=x^{i}-v_{i} t$, and $t_{G}=t$, the Minkowskian line element takes the form

$$
\mathrm{d} s^{2}=\left(c^{2}-v^{2}\right) \mathrm{d} t_{G}^{2}-2 v_{i} \mathrm{~d} x_{G}^{i} \mathrm{~d} t_{G}-\delta_{i j} \mathrm{~d} x_{G}^{i} \mathrm{~d} x_{G}^{j} .
$$

It will now be convenient to set $x^{0}=c t, \quad \beta_{i}=v_{i} / c$, and to write (25) and (27) as follows:

$\mathrm{d} s^{2}=g_{\mu v}^{\prime}\left(\beta_{i}\right) \mathrm{d} x^{\prime \mu} \mathrm{d} x^{\prime \nu}$, and $\mathrm{d} s^{2}=g_{\mu v}^{G}\left(\beta_{i}\right) \mathrm{d} x_{G}^{\mu} \mathrm{d} x_{G}^{v}$, with $(\mu, v=0,1, \cdots, n)$. One then finds, omitting the arguments, that

$$
g_{\mu \lambda}^{\prime} g_{\lambda v}^{G}=\delta_{\mu \nu},
$$

so that the Galilean metric tensor is the inverse of EST metric tensor, and hence is of same form as the contravariant EST metric tensor $g^{\prime \mu \nu}\left(\beta_{i}\right)$ which is needed to construct the D' Alembertian operator under the EST, consequently

$$
g^{\prime \mu v} \partial_{\mu}^{\prime} \partial_{v}^{\prime}=g_{\mu \nu}^{G} \partial_{\mu}^{\prime} \partial_{v}^{\prime} .
$$

This result was given in [1] without explanation, but subsequently it has been found that an algebraic analysis provides insight as to why the GT appears, when use is made of another finding from [1]: the LT, represented as a matrix, can be factored into a product of three unimodular, non-commuting matrices. In [1], this was done for the velocity along one of the coordinate axes, but the result holds more generally. Also, although the matrices there were for only three spatial dimensions, the factorization holds in $n$ spatial dimensions. Thus, if the space-time coordinates in the rest frame are described by the column vector $\boldsymbol{X}=\left(x_{0}, x_{1}, \cdots, x_{n}\right)$, and the transformed coordinates in the Lorentz-boosted frame by the column vector $\boldsymbol{X}_{L}=\left(x_{L 0}, x_{L 1}, \cdots, x_{L n}\right)$, the LT can be written

$$
\boldsymbol{X}_{L}=\boldsymbol{L}\left(\beta_{i}\right) \boldsymbol{X} .
$$

The matrix $\boldsymbol{L}\left(\beta_{i}\right)$ is the $(1+n)$ square matrix that represents the $\mathrm{LT}$, which in turn can be written

$$
\boldsymbol{L}\left(\beta_{i}\right)=\boldsymbol{E}\left(\beta_{i}\right) \boldsymbol{F}\left(\beta_{i}\right) \boldsymbol{G}\left(\beta_{i}\right),
$$

where $\boldsymbol{E}\left(\beta_{i}\right), \boldsymbol{F}\left(\beta_{i}\right), \boldsymbol{G}\left(\beta_{i}\right)$ are all unimodular, non-commuting matrices. The matrix $\boldsymbol{G}\left(\beta_{i}\right)$ represents the standard Galilean transformation with $\boldsymbol{G}\left(-\beta_{i}\right)=\boldsymbol{G}^{-1}\left(\beta_{i}\right)$, in keeping with its group property. The matrix $\boldsymbol{F}\left(\beta_{i}\right)$ is symmetric $F_{\mu \nu}=F_{v \mu}$, with $F_{00}=\gamma^{-1}, \quad F_{i o}=F_{o i}=0$, and $F_{i j}=\delta_{i j}+\beta_{i} \beta_{j}(\gamma-1) \beta^{-2}$, with $\beta^{2}=\beta_{i} \beta_{i}$. Since $\boldsymbol{F}\left(\beta_{i}\right)$ contains the time dilation and contraction factors, which are even functions of the velocity, consequently $\boldsymbol{F}\left(-\beta_{i}\right)=\boldsymbol{F}\left(\beta_{i}\right)$. The matrix $\boldsymbol{E}\left(\beta_{i}\right)$ represents the local time transformation, and analogous to the GT, $\boldsymbol{E}\left(-\beta_{i}\right)=\boldsymbol{E}^{-1}\left(\beta_{i}\right)$, so that, together with its multiplicative properties, the local time transformations form a group as do the GTs, indeed, $\boldsymbol{E}\left(\beta_{i}\right)$ is the transpose of $\boldsymbol{G}\left(\beta_{i}\right), \boldsymbol{E}\left(\beta_{i}\right)=\tilde{\boldsymbol{G}}\left(\beta_{i}\right)$. Then, omitting the arguments of the matrices, (30) may be written as

$$
\boldsymbol{L}=\tilde{\boldsymbol{G}} \boldsymbol{F} \boldsymbol{G} .
$$

Since $\boldsymbol{X}^{\prime}=\boldsymbol{E}^{-1} \boldsymbol{X}_{L}$, the equation for the EST that follows from (30) and (32) is

$$
\boldsymbol{X}^{\prime}=\boldsymbol{F} \boldsymbol{G X}=\tilde{\boldsymbol{G}}^{-1} \mathbf{L} \boldsymbol{X},
$$

and hence $\boldsymbol{X}=\boldsymbol{L}^{-1} \tilde{\boldsymbol{G}} \boldsymbol{X}^{\prime}$ and $\tilde{\boldsymbol{X}}=\tilde{\boldsymbol{X}}{ }^{\prime} \tilde{\boldsymbol{L}}^{-1}$. The quadratic form in the rest frame that corresponds to the Minkowski line element, and in which the diagonal matrix $\boldsymbol{M}=\operatorname{diag}(1,-1, \cdots,-1)$ corresponds to the Minkowski metric tensor, is given by $\tilde{\boldsymbol{X}} \boldsymbol{M} \boldsymbol{X}$, so that after transforming to the primed frame, and using the above expressions for $\boldsymbol{X}$ and $\tilde{\boldsymbol{X}}$, one has

$$
\tilde{\boldsymbol{X}} \boldsymbol{M} \boldsymbol{X}=\tilde{\boldsymbol{X}}^{\prime} \boldsymbol{G} \tilde{\boldsymbol{L}}^{-1} \boldsymbol{M} \boldsymbol{L}^{-1} \tilde{\boldsymbol{G}} \boldsymbol{X}^{\prime} .
$$

Since the inverse of an LT is an LT, and the LTs leave the Minkowski line element invariant, and consequently 
the corresponding quadratic form, one has $\tilde{\boldsymbol{L}}^{-1} \boldsymbol{M} \boldsymbol{L}^{-1}=\boldsymbol{M}$. It follows that $\tilde{\boldsymbol{X}} \boldsymbol{M} \boldsymbol{X}=\tilde{\boldsymbol{X}}^{\prime} \boldsymbol{G} \boldsymbol{M} \tilde{\boldsymbol{G}} \boldsymbol{X}^{\prime}$, and hence the matrix associated with the metric tensor for the EST frame is given by $\boldsymbol{G M} \tilde{\boldsymbol{G}}$, so that

$$
\boldsymbol{G M} \tilde{\boldsymbol{G}} \Leftrightarrow\left\|g_{\mu \nu}^{\prime}\right\| \text {. }
$$

For the GT, $\boldsymbol{X}_{G}=\boldsymbol{G} \boldsymbol{X}, \boldsymbol{X}=\boldsymbol{G}^{-1} \boldsymbol{X}_{G}, \tilde{\boldsymbol{X}}=\tilde{\boldsymbol{X}}_{G} \tilde{\boldsymbol{G}}^{-1}$, hence $\tilde{\boldsymbol{X}} \boldsymbol{M} \boldsymbol{X}=\tilde{\boldsymbol{X}}_{G} \tilde{\boldsymbol{G}}^{-1} \boldsymbol{M} \boldsymbol{G}^{-1} \boldsymbol{X}_{G}$, so that

$$
\tilde{\boldsymbol{G}}^{-1} \boldsymbol{M G}^{-1} \Leftrightarrow\left\|g_{\mu \nu}^{G}\right\|
$$

Then since $\boldsymbol{M}^{2}=\boldsymbol{I}$, where $\boldsymbol{I}$ is the identity, one has

$$
\boldsymbol{G} \boldsymbol{M} \tilde{\boldsymbol{G}} \tilde{\boldsymbol{G}}^{-1} \boldsymbol{M} \boldsymbol{G}^{-1}=\boldsymbol{G} \boldsymbol{M}^{2} \boldsymbol{G}^{-1}=\boldsymbol{I},
$$

and hence the relation given in (28) follows.

The above formalism enables one to obtain the PGT very readily. One goes back to (33), and upon restoring the arguments for both $\boldsymbol{F}$ and $\boldsymbol{G}$, it becomes, $\boldsymbol{X}^{\prime}=\boldsymbol{F}\left(\beta_{i}\right) \boldsymbol{G}\left(\beta_{i}\right) \boldsymbol{X}$, and similarly $\boldsymbol{X}^{\prime \prime}=\boldsymbol{F}\left(-\beta_{i}\right) \boldsymbol{G}\left(-\beta_{i}\right) \boldsymbol{X}$, and because $\boldsymbol{F}\left(-\beta_{i}\right)=\boldsymbol{F}\left(\beta_{i}\right)$, and also, $\boldsymbol{G}\left(-\beta_{i}\right)=\boldsymbol{G}^{-1}\left(\beta_{i}\right)$ hence $\boldsymbol{X}=\boldsymbol{G}\left(\beta_{i}\right) \boldsymbol{F}^{-1}\left(\beta_{i}\right) \boldsymbol{X}^{\prime \prime}$, so that

$$
\boldsymbol{X}^{\prime}=\boldsymbol{F}\left(\beta_{i}\right) \boldsymbol{G}^{2}\left(\beta_{i}\right) \boldsymbol{F}^{-1}\left(\beta_{i}\right) \boldsymbol{X}^{\prime \prime}=\boldsymbol{F}\left(\beta_{i}\right) \boldsymbol{G}\left(2 \beta_{i}\right) \boldsymbol{F}^{-1}\left(\beta_{i}\right) \boldsymbol{X}^{\prime \prime},
$$

after using, $G^{2}\left(\beta_{i}\right)=G\left(2 \beta_{i}\right)$ because of the group property of the GT. One next uses the readily verified relation $\boldsymbol{F}\left(\beta_{i}\right) \boldsymbol{G}\left(2 \beta_{i}\right)=\boldsymbol{G}\left(2 \beta_{i} \gamma^{2}\right) \boldsymbol{F}\left(\beta_{i}\right)$, followed by $\boldsymbol{F}\left(\beta_{i}\right) \boldsymbol{F}^{-1}\left(\beta_{i}\right)=\boldsymbol{I}$ to obtain the $1+n$ dimensional matrix form of (21) and (22) for the PGT

$$
\boldsymbol{X}^{\prime}=\boldsymbol{G}\left(2 \beta_{i} \gamma^{2}\right) \boldsymbol{X}^{\prime \prime}
$$

It will next be shown that between any two inertial frames in relative motion, there always exists a pseudoGalilean transformation connecting them. Choose one of the frames $S^{\prime \prime}$ to be initially the rest frame, and orient its coordinate system so that, say, the positive $x^{\prime \prime}$-axis is in the direction of the velocity of the other frame $S^{\prime}$. Next, introduce a third inertial frame $S$ that will be described as the center-of-velocity frame. In this frame clocks are synchronized so that the speed of light is $c$ in all directions. To find the equivalent of the velocity $v$ of $S^{\prime}$ with respect to $S$ that was used to obtain the PGT, assume that a set of clocks are internally synchronized in $S^{\prime \prime}$ so that the speed of light in all directions is $c$, and that measured with these clocks, the velocity of $S^{\prime}$ relative to $S^{\prime \prime}$ along the $x^{\prime \prime}$-axis is $w$. The velocity of $S$ is chosen to be parallel to $w$, and has the value $v$ relative to $S^{\prime \prime}$, while $v$ is chosen so that the velocity of $S^{\prime}$ with respect to $S$ is also $v$. Using the expression for relativistic relative velocity, these conditions require that

$$
v=\frac{w-v}{1-\left(w v / c^{2}\right)} .
$$

The solution to the resulting quadratic equation for $v$ that leads to the pre-relativistic solution for $w / c \ll 1$ is given by

$$
v=c^{2} w^{-1}\left(1-\sqrt{1-(w / c)^{2}}\right) .
$$

Upon expanding to first order, one has $v=w / 2$ as required, which can also be inferred from (40) by setting $c=\infty$. As discussed above, when clocks in $S^{\prime}$ and $S^{\prime \prime}$ are synchronized externally with those in $S$, there is a PGT connecting them.

\section{Validity of the PGT for Stellar Aberration}

It is interesting to show how the PGT may be used to give an alternative treatment of stellar aberration that agrees with the prediction of SR and the LT. Before showing this, it is important to note that the EST should agree with the LT since the spatial coordinates for the two transformations are the same, and since all one measures in aberration is an angle, and since the tangent of the angle is the ratio of the spatial displacements, the result must be the same for the two transformations, even though the light travels with different velocities for the LT and EST observers. In the comparable analysis of Puccini and Selleri [49], the EST is referred to as the "inertial transformation." Unlike the interpretation of Puccini and Selleri, however, the interpretation here is based solely on external synchronization, rather than "absolute motion," although the latter concept motivated [1]. 
As indicated in Section 1, considerations about the ether are omitted here for brevity, but given in [15] [32] which reference Einstein's [50] later support for an ether, since according to general relativity space has physical properties. However, because of the special relativity principle, for a wide range of experiments, reference to the ether is unnecessary in the theoretical analysis.

The aberration will be worked out first for the LT, in the notation used previously, so as to facilitate comparison. The light source and the observer will be taken to be in the $x-y$ plane, with the observer at rest, and the source traveling in the negative $x$-direction with velocity $-w$ relative to the observer. Then with frame $S^{\prime}$ that of the observer, and frame $S^{\prime \prime}$ that of the source, one has, $\mathrm{d} x_{L}^{\prime}=\gamma(w)\left(\mathrm{d} x_{L}^{\prime \prime}-w \mathrm{~d} t_{L}^{\prime \prime}\right), \mathrm{d} y_{L}^{\prime}=\mathrm{d} y_{L}^{\prime \prime}$ where the space-time coordinates in both frames are assumed to be Lorentzian, and hence the metrics are Minkowskian. Assume that the light leaves the star traveling in the negative $y^{\prime \prime}$ direction, then $\mathrm{d} x_{L}^{\prime \prime}=0$, so that $\mathrm{d} x_{L}^{\prime}=-\gamma(w) w \mathrm{~d} t_{L}^{\prime \prime}$, and $\mathrm{d} y_{L}^{\prime}=\mathrm{d} y_{L}^{\prime \prime}=-c \mathrm{~d} t_{L}^{\prime \prime}$. The tangent of the aberration angle $\theta_{L}$ is given by $\tan \theta_{L}=\mathrm{d} x_{L}^{\prime} / \mathrm{d} y_{L}^{\prime}=\gamma(w) w / c$, and hence

$$
\theta_{L}=\tan ^{-1}(\gamma(w) w / c) .
$$

This is the standard SR expression for stellar aberration, and note, importantly, that in computing the tangent, the time interval $\mathrm{d} t_{L}^{\prime \prime}$ cancels out, so the SR expression for the aberration does not involve the relativity of simultaneity, and hence holds for the EST as well. If one now uses the PGT, then from Equation (13),

$\mathrm{d} x^{\prime}=\mathrm{d} x^{\prime \prime}-u \mathrm{~d} t^{\prime \prime}, \mathrm{d} y^{\prime}=\mathrm{d} y^{\prime \prime}$, with $u=2 v \gamma^{2}(v)$ from (15), and therefore, with the same assumptions as above, $\mathrm{d} x^{\prime \prime}=0$, so that $\mathrm{d} x^{\prime}=-u \mathrm{~d} t^{\prime \prime}, \mathrm{d} y^{\prime}=\mathrm{d} y^{\prime \prime}=-c \mathrm{~d} t^{\prime \prime}$, one obtains $\theta^{\prime}=\tan ^{-1}(u / c)$, which is of the same form as in Newtonian physics. However, after substituting the value of $u$ from (15), one has

$$
\theta^{\prime}=\tan ^{-1}\left(2 v \gamma^{2} / c\right)
$$

Since $v$ is the earth's velocity relative to the center-of-velocity frame, and $-v$ the velocity of the star relative to that frame, the relativistic relative velocity $w$ of the earth with respect to the star in terms of $v$ is

$$
w=2 v /\left(1+(v / c)^{2}\right)
$$

which is another form of (40). After introducing $w(v)$ into (42) for $\theta_{L}$, one has

$$
\theta_{L}=\tan ^{-1}\left(\gamma(w(v)) 2 v /\left(1+(v / c)^{2}\right) c\right)=\tan ^{-1}\left(2 v \gamma^{2}(v) / c\right)=\theta^{\prime} .
$$

This establishes consistency between the PGT and the LT for stellar aberration.

\section{Application of the PGT to the Doppler Effect}

The Doppler effect will be calculated for the PGT for motion along the $x$-axis and will be shown to reduce to the standard expression based on the LT. The notation will be the same as above. When dealing with the EST or PGT, or non-Lorentz transformations more generally, there can be significant differences between the covariant and contravariant components of tensors, since more than a minus sign is usually involved. Thus, in order to relate the PGT expression for the Doppler effect with that using the LT, equivalent expressions for frequency must be used, albeit in the PGT case, expressed as a function of $v$, while in the LT case, expressed as a function of $w$, also there has to be agreement as to the direction in which the light is being observed. To obtain equivalent expressions for frequency, it is helpful to note that the phase $\Phi$ of the wave in the center-of-velocity frame $S$ is given by $\Phi=p_{\mu} x^{\mu} / \hbar$, where $p_{\mu}$ are the covariant components of the photon's energy-momentum four-vector for which the frequency $f$ is given by $f=p_{0} c / h$. As shown in [1], and repeated here for convenience, under the local time transformation, the time component of the covariant form of the LT energy-momentum four-vector $p_{L 0}^{\prime}$ is invariant upon transforming to the time component of the EST covariant four-momentum $p_{0}^{\prime}$, since

$$
p_{0}^{\prime}=p_{L \mu}^{\prime} \partial x_{L}^{\prime \mu} / \partial x^{\prime 0}
$$

and since $x^{\prime 0}=x_{L}^{\prime 0}+\beta x_{L}^{\prime 1}$, and $x_{L}^{\prime i}=x^{\prime i}$, then $\partial x_{L}^{\prime i} / \partial x^{\prime 0}=0$, and $\partial x_{L}^{\prime 0} / \partial x^{\prime 0}=1$. Consequently, $p_{0}^{\prime}=p_{L 0}^{\prime}$, and likewise, for the frame $S^{\prime \prime}, p_{0}^{\prime \prime}=p_{L 0}^{\prime \prime}$, and hence the corresponding frequencies in the frames $S^{\prime}$ and $S^{\prime \prime}$ satisfy

$$
f^{\prime}=f_{L}^{\prime}, f^{\prime \prime}=f_{L}^{\prime \prime}
$$


The expressions for $f^{\prime}$ and $f^{\prime \prime}$ will be obtained by transforming the phase as given in $S$, when the light is assumed to be traveling in the $z=0$ plane. Then, since $p_{x} / \hbar=-2 \pi f \cos \theta / c, \quad p_{y} / \hbar=-2 \pi f \sin \theta / c$, the phase in $S$ is given by the standard expression $\Phi=2 \pi f(t-(x \cos \theta+y \sin \theta) / c)$. Since $\Phi$ is invariant when transforming to $S^{\prime}$ and $S^{\prime \prime}$, upon introducing the inverse of the EST from $S$ to $S^{\prime}$, given in (3) and (4), into the phase, with comparable equations holding for $S$ to $S^{\prime \prime}$ after setting $v \rightarrow-v$, one obtains $f^{\prime}=f \gamma(1-\beta \cos \theta)$, and $f^{\prime \prime}=f \gamma(1+\beta \cos \theta)$, and hence

$$
f^{\prime}=f^{\prime \prime} \frac{(1-\beta \cos \theta)}{(1+\beta \cos \theta)}
$$

To express $\cos \theta$ in terms of $\cos \theta^{\prime}$, it is convenient to use the local time transformation in differential form, $\mathrm{d} t^{\prime}=\mathrm{d} t_{L}^{\prime}+(\beta / c) \mathrm{d} x_{L}^{\prime}$ so that

$$
\frac{\mathrm{d} x^{\prime}}{\mathrm{d} t^{\prime}}=\frac{\mathrm{d} x_{L}^{\prime} / \mathrm{d} t_{L}^{\prime}}{\left(1+(\beta / c) \mathrm{d} x_{L}^{\prime} / \mathrm{d} t_{L}^{\prime}\right)}=\frac{c \cos \theta^{\prime}}{\left(1+\beta \cos \theta^{\prime}\right)}
$$

in which $\cos \theta_{L}^{\prime}=\cos \theta^{\prime}$ has been used, since $x^{\prime i}=x_{L}^{\prime i}$. From (3) and (4), it follows that $\mathrm{d} x / \mathrm{d} t=\gamma^{-2}\left(\mathrm{~d} x^{\prime} / \mathrm{d} t^{\prime}\right)+v$, and since $\mathrm{d} x / \mathrm{d} t=c \cos \theta$, one has

$$
\cos \theta=\frac{\gamma^{-2} \cos \theta^{\prime}}{1+\beta \cos \theta^{\prime}}+\beta .
$$

This somewhat lengthy analysis to obtain the relation between $\cos \theta$ and $\cos \theta^{\prime}$ was used because the speed of light in $S^{\prime}$ is not $c$ in all directions, and hence $\mathrm{d} x^{\prime} / \mathrm{d} t^{\prime} \neq c \cos \theta^{\prime}$, as follows from (49). Finally, upon substituting the above expression for $\cos \theta$ into (48), and reducing, one obtains the PGT expression for the Doppler effect

$$
f^{\prime}=f^{\prime \prime} \frac{\left(1-\beta^{2}\right)}{1+2 \beta \cos \theta^{\prime}+\beta^{2}} .
$$

After one sets $f^{\prime}=f_{L}^{\prime}, f^{\prime \prime}=f_{L}^{\prime \prime}$, and $w / c=2 \beta /\left(1+\beta^{2}\right)$, that follow from (47) and (44), and uses $\cos \theta^{\prime}=\cos \theta_{L}^{\prime}$, and $\sqrt{1-(w / c)^{2}}=\left(1-\beta^{2}\right) /\left(1+\beta^{2}\right)$, the equation reduces to

$$
f_{L}^{\prime}=f_{L}^{\prime \prime} \frac{\sqrt{1-(w / c)^{2}}}{1+(w / c) \cos \theta_{L}^{\prime}}
$$

which is the standard expression for the relativistic Doppler effect based on the LT. This again establishes consistency between the PGT and the LT.

\section{One-Way Speed of Light, Simultaneity, Complementarity, and General Covariance}

The fact that the one-way speed of light is not invariant under the EST, while simultaneity between the two inertial frames does remain invariant, and in contrast, the one-way speed of light is invariant under the LT, while simultaneity is no longer invariant, but becomes relative, can be seen as a manifestation of a complementarity between maintaining invariance of the one-way speed of light and maintaining invariance of simultaneity in the same transformation. This situation provides an illustration of Bohr's [51] idea that the complementarity principle has a wider range of application than in a purely quantum mechanical context. It is interesting to note that if the speed of light were infinite, this complementarity between invariance of the one-way speed of light and invariance of simultaneity would be eliminated, which parallels the fact that the complementarity between position and momentum would be eliminated if Planck's constant vanished. This limiting reciprocal behavior of the speed of light and the quantum of action, that would eliminate both complementary relations, raises the question as to whether they are independent constants of nature, as in current theory, or whether at a deeper level they emerge together from a more fundamental theory? An answer to this question could possibly provide a new path towards the unification of gravitation and quantum theory, since an inverse relation between $\hbar$ and $c$ in- 
volving the Newtonian gravitational constant is readily obtained by rewriting the standard expression for the square of the Planck mass, $m_{p}$, as $\hbar c=G m_{P}^{2}$. However, since the fine-structure constant $\alpha$ may be written $\hbar c=e^{2} \alpha^{-1}$, such unification may also require unifying gravitation and electromagnetism as well. A further consequence of this new complementarity is that it works against Kretschmann's [52] view that general covariance does not have physical significance, since his view misses the fact that general covariance allows the EST that keeps simultaneity invariant, instead of the invariance of the one-way speed of light, in contrast with the LT that does the opposite, and also it allows the PGT, which leaves lengths, clock rates, and simultaneity invariant, albeit not the one-way speed of light, again in contrast to the LT. Although Kretschman's view is the prevailing one [3] [5], and is one with which Einstein concurred [53], while emphasizing the principle's heuristic value, Rindler [4] notes that additional conditions may give physical content to the principle, and he gives reference to the work of Anderson [54], who, like the author, believes that the principle does have physical significance. However, Anderson's view is based on the principle of equivalence, Einstein's field equations, simplicity, and aesthetic considerations, rather than, as here, on the two physically different methods of synchronization that the principle allows, and the complementarity that results. For an argument by the author involving commutators, that supports the physical significance of general covariance in the quantum domain, see [55].

\section{Concluding Remarks}

The preceding analysis shows that, in accordance with the principle of general covariance, one can consistently use a linear transformation between two inertial frames based on an external synchronization that keeps simultaneity invariant, but not the one-way speed of light, while keeping the out-and-back speed invariant. This external synchronization leads to the EST, and contrasts with the LT, based on internal synchronization. Also, as was shown in Section 4, the EST enables one to set up an exact analogy with the Sagnac effect, for a circular path in a rotating frame, both in a vacuum and in a dielectric medium, again in contrast with the LT. However, the main finding of the present study is the pseudo-Galilean transformation (PGT), as discussed in Sections 5 - 7. As discussed in Section 8, there is a complementarity between maintaining invariance of the one-way speed of light, and maintaining invariance of simultaneity in the same linear transformation, that supports the physical significance of general covariance.

Future applications of the PGT might be to the decay of a particle into two particles of equal mass, since the parent particle's rest frame is the center-of-velocity frame. Also, the PGT might lead to simplifications in analyzing colliding beam experiments between particles of the same mass, since the laboratory, ideally, would be the center-of-velocity frame, and the colliding beams could be related by the PGT, so that observers traveling with either colliding particle, would not see the other particle, say a proton in the LHC, as "pancaked.” But whether these applications will lead to any simplifications in the study of particle decays and collisions will require further study.

A preliminary version of this work was presented at the 2011 Anaheim, California meeting of the American Physical Society [56].

I would like to acknowledge very helpful and stimulating correspondence with Dr. Gregory B. Malykin of the Russian Academy of Sciencs in Nizhny Novgorod, and also for sending me a copy of Dr. Franz Harress' dissertation. The author is also indebted to Prof. Eric Sheldon for reference [38], and for his interest in my studies of relativistic transformations.

\section{References}

[1] Tangherlini, F.R. (1958) The Velocity of Light in Uniformly Moving Frames. Ph.D. Dissertation, Stanford University, Stanford, Published in: (2009) Abraham Zelmanov Journal, 2, 44-110. http://zelmanov.ptep-online.com/papers/zj-2009

[2] Stachel, J. (1989) Einstein's Search for General Covariance. In: Howard, D. and Stachel, J., Eds., Einstein and the History of General Relativity, Birkhäuser, Boston, 63-100.

[3] Pauli, W. (1958) Theory of Relativity. Pergamon Press, Oxford, 149-150.

[4] Rindler, W. (1969) Essential Relativity. Van Nostrand Reinhold, Co., 196-197.

[5] Weinberg, S. (1972) Gravitation and Cosmology. John Wiley \& Sons, Inc., Hoboken, 91-93.

[6] Tangherlini, F.R. (2008) Physica Scripta, 77, Article ID: 065008 (9 pages). 
[7] Feinberg, G. (1967) Physical Review, 159, 1089-1105. http://dx.doi.org/10.1103/PhysRev.159.1089

[8] Schmidt, H. (1958) Zeitschrift für Physik, 151, 365-374. http://dx.doi.org/10.1007/BF01338390

[9] Schmidt, H. (1958) Zeitschrift für Physik, 151, 408-420. http://dx.doi.org/10.1007/BF01337942

[10] Bilaniuk, O.M.P., Deshpande, V.K. and Sudarshan, E.C.G. (1962) American Journal of Physics, 30, 718-723. http://dx.doi.org/10.1119/1.1941773

[11] Tanaka, S. (1960) Progress of Theoretical Physics, 24, 171-200. http://dx.doi.org/10.1143/PTP.24.171

[12] Terletski, Ya. (1961) Soviet Physics-Doklady, 5, 782-785.

[13] Recami, E. (1986) La Rivista Del Nuovo Cimento Series 3, 9, 1-178. http://dx.doi.org/10.1007/BF02724327

[14] Fröman, O. (1994) Archive Hist. Exact Sciences, 48, 373-373. http://dx.doi.org/10.1007/BF00375087

[15] Tangherlini, F.R. (1994) Il Nuovo Cimento B Series 11, 109, 929-951. http://dx.doi.org/10.1007/BF02726141

[16] Tangherlini, F.R. (1961) Supplemento del Nuovo Cimento, 20, 1-86; Chinese transl.: Zhu, P.Y. (1963) Shanghai Science and Technology Press, Shanghai.

[17] Mansouri, R. and Sexl, R.U. (1977) General Relativity and Gravitation, 8, 497-513; 515-524; 809-814. http://dx.doi.org/10.1007/BF00762634

[18] Chang, T. (1979) Physics Letters A, 70, 1-2. http://dx.doi.org/10.1016/0375-9601(79)90309-8

[19] Tangherlini, F.R. (2009) Abraham Zelmanov Journal, 2, 111-120.

[20] Sjödin, T. (1979) Il Nuovo Cimento B Series 11, 114, 229-246. http://dx.doi.org/10.1007/BF02743433

[21] Cavalleri. G. and Spinelli, G. (1983) Foundations of Physics, 13, 1221-1229. http://dx.doi.org/10.1007/BF00727994

[22] Spavieri, G. (1986) Physical Review A, 34, 1708-1713. http://dx.doi.org/10.1103/PhysRevA.34.1708

[23] Selleri, F. (1996) Foundations of Physics, 26, 641-664. http://dx.doi.org/10.1007/BF02058237

[24] Selleri, F. (2005) Foundations of Physics Letters, 18, 325-339. http://dx.doi.org/10.1007/s10702-005-7123-8

[25] Cavalleri, G. and Tonni, E. (1998) Physical Review E, 57, 3478-3484. http://dx.doi.org/10.1103/PhysRevE.57.3478

[26] Tonni, E. (1999) Nuovo Cimento della Societa Italiana di Fisica [Sezione] B, 114, 561-568.

[27] Cavalleri, G., Tonni, E. and Barbareo, F. (2013) Physical Review E, 87, Article ID: 043202 (13 pages).

[28] Malykin, G.B. and Malykin, E.G. (2009) Abraham Zelmanov Journal, 2, 121-124.

[29] Eagle, A. (1938) Philosophical Magazine, 26, 410-414.

[30] Eagle, A. (1939) Philosophical Magazine, 28, 592-595.

[31] Eagle, A. (1939) Philosophical Magazine Series 6, 28, 694-701.

[32] Tangherlini, F.R. (2009) Abraham Zelmanov Journal, 2, 29-43.

[33] Reichenbach, H. (1928) Philosphie der Raum-Zeit-Lehre. Walter Gruyter and Co., Berlin; English Translation, Reichenbach, M. and Freud, J. (1958) The Philosophy of Space and Time. Dover, New York, 123-129.

[34] Anderson, R., Vetharaniam, J. and Stedman, G.E. (1998) Physics Reports, 295, 93-180. http://dx.doi.org/10.1016/S0370-1573(97)00051-3

[35] Smoot, G.F., Bennett, C.L., Kogut, A., et al. (1992) Astrophysics Journal, 396, L1-L5. http://dx.doi.org/10.1086/186504

[36] Conklin, E.K. (1969) Nature, 122, 971-972.

[37] Henry, P.S. (1971) Nature, 231, 516-518. http://dx.doi.org/10.1038/231516a0

[38] Wolf, P. and Petit, G. (1997) Physical Review A, 56, 4405-4409. http://dx.doi.org/10.1103/PhysRevA.56.4405

[39] Sagnac, G. (1913) Comptes Rendus, 157, 708-710.

[40] Sagnac, G. (1913) Comptes Rendus, 157, 1410-1413.

[41] Post, E.J. (1967) Reviews of Modern Physics, 39, 475-481. http://dx.doi.org/10.1103/RevModPhys.39.475

[42] Anderson, R., Bilger, H.R. and Stedman, G.E. (1994) American Journal of Physics, 62, 975-985. http://dx.doi.org/10.1119/1.17656

[43] Malykin, G.B. (2000) Physics-Uspekhi, 43, 1229-1252. http://dx.doi.org/10.1070/PU2000v043n12ABEH000830

[44] Ghosal, S.K., Raychaudhuri, B., Chowdhury, A.K. and Sarker, M. (2004) Foundations of Physics Letters, 17, 457-477. http://dx.doi.org/10.1023/B:FOPL.0000042698.27763.0b

[45] Harzer, P. (1914) Astronomische Nachrichten, 198, 377-392.

[46] Harress, F. (1911) Die Geschwindigkeit des Lichtes in bewegten Körpern. Ph.D. Dissertation, Jena University, Jena, 
unpublished.

[47] Arditty, H.J. and Lefèvre, H.C. (1981) Optics Letters, 6, 401-403. http://dx.doi.org/10.1364/OL.6.000401

[48] Bridgman, P.W. (1962) A Sophisticates Primer of Relativity. Wesleyan University Press, Middletown, 92-93.

[49] Puccini, G.D. and Selleri, F. (2002) Nuovo Cimento B, 117, 283-293.

[50] Einstein, A. (1920) Äether und Relativitätstheorie. Springer, Berlin; English Translation: Harris, A. (1953) In: Essays in Science. Philosophical Library, New York, 98-111.

[51] Bohr, N. (1961) Atomic Physics and Human Knowledge. Science Editions, New York, 67-82.

[52] Kretschmann, E. (1917) Annalen der Physik, Leipzig, 53, 575-614.

[53] Einstein, A. (1918) Annalen der Physik, Leipzig, 55, 241-244. http://dx.doi.org/10.1002/andp.19183600402

[54] Anderson, J.L. (1967) Principles of Relativity Physics. Academic Press, New York, 338-340.

[55] Tangherlini, F.R. (1970) Lettere al NuovoCimento, Series I, 2, 88-92.

[56] Tangherlini, F.R. (2011) Bulletin of the American Physical Society, 56, 59. 